\title{
Emacs-reveal: A software bundle to create OER presentations
}

\author{
Jens Lechtenbörger ${ }^{1}$ \\ 1 ERCIS, University of Münster, Germany
}

DOI: $10.21105 /$ jose. 00050

\section{Software}

- Review ct

- Repository ca

- Archive ¿

Submitted: 18 April 2019

Published: 04 August 2019

\section{License}

Authors of papers retain copyright and release the work under a Creative Commons Attribution 4.0 International License (CC-BY).

\section{Introduction}

According to the Ljubljana OER Action Plan 2017 (UNESCO, 2017), "Open Educational Resources (OER) support quality education that is equitable, inclusive, open and participatory." However, several challenges are known that hinder widespread creation, use, and re-use of OER. The first challenge identified in the Action Plan lies in "the capacity of users to find, re-use, create, and share OER", and the first action category addressing that challenge, "Building awareness and skills to use OER", lists ten actions, among which action (c) reads as follows: "Disseminate the findings of research on OER to support models of good practice with a focus on cost-effectiveness, sustainability, exploration of new tools and technologies for the creation and sharing of OER".

Emacs-reveal is a Free/Libre and Open Source Software (FLOSS) bundle (combining novel and established FLOSS components) in accordance with action (c). Briefly, emacsreveal is a software tool, embedded in a powerful Continuous Integration infrastructure, to create and (re-) use OER slideshows (for courses and talks) with references and embedded multimedia contents such as figures, audio explanations, animations, videos, quizzes, and live code execution. The software simplifies creation and re-use of OER by addressing OER-specific requirements as summarized next.

\section{Technical requirements for OER}

For educational resources to be free and open, next to proper licensing requirements also technical requirements exist (as defined in the ALMS framework, which was proposed by Hilton, Wiley, Stein, \& Johnson (2010) and extended by Lechtenbörger (2019a)):

- OER should be usable (for learning) with FLOSS on (almost) any device, also mobile and offline.

- OER should be editable with FLOSS (this requires source file access).

- OER should be re-usable under the Single Sourcing paradigm (Rockley, 2001), which enables reuse and revision from a single, consistent source without copy\&paste (copy\&paste creates isolated copies, where the reconciliation of changes and improvements by different individuals would be almost impossible).

- OER should offer a separation of contents from layout (then, experts for content do not need to be design experts as well; also, cross-organizational collaboration is supported where each organization can apply its own design guidelines).

- OER should be defined in a lightweight markup language, which is easy to learn and which enables the use of industrial-strength version control systems such as Git for the management of OER collaboration (comparison, revision, merge). 


\section{Statement of Need}

The author was unable to locate FLOSS for the creation of OER presentations with audio explanations that satisfies the above requirements, confirming the need for "new tools and technologies for the creation and sharing of OER" identified in the Action Plan cited above (UNESCO, 2017).

For example, support for presentations created with software such as LibreOffice Impress on mobile devices was and still is limited, as is support for Single Sourcing and separation of contents from layout. Beamer LaTeX presentations (see Wright, 2019) support Single Sourcing with separation of contents from layout, and generated PDF documents can embed audio files, but their playback using FLOSS on mobile devices was and still is limited. The author did not systematically analyze Wiki-style presentations, such as those created with SlideWiki (Auer, Khalili, \& Tarasowa, 2013), because he was looking for support for Single Sourcing and collaboration based on the capabilities of decentralized version control systems such as Git (Chacon \& Straub, 2014).

\section{Functionality of emacs-reveal}

Emacs-reveal meets all of the above requirements, which lowers entry barriers towards a more widespread creation of OER. As described in Lechtenbörger (2019b), for OER creators it simplifies licensing attribution when re-using figures with machine-readable meta-data based on an extension of CC REL (The Creative Commons Rights Expression Language, see Abelson, Adida, Linksvayer, \& Yergler (2012)), (a) avoiding manual identification and copying of licensing information, which is among the most time-consuming factors for OER projects (see Falconer, Littlejohn, McGill, \& Beetham, 2016), and (b) making licensing information accessible on the Semantic Web with RDFa in HTML (see Horrocks, 2008 for a general introduction to the Semantic Web).

With emacs-reveal, source files for presentations are written in the lightweight markup language Org Mode (Schulte \& Davison, 2011) and converted to slideshows based on the HTML presentation framework reveal.js. Org mode is native to the text editor GNU Emacs but can be edited in any text editor; it is also used in other contexts to create modular and reusable teaching materials (Ro \& Namkoong, 2019). Docker images for emacs-reveal can be used to generate OER presentations in GitLab Continuous Integration infrastructures, notably a Howto presentation for emacs-reveal is maintained that way, as are presentations for a course on Operating Systems (based on Just-in-Time Teaching (Novak, 2011), which relies on considerable self-study) for which emacs-reveal was originally developed.

\section{Acknowledgements}

The author acknowledges funding for a fellowship for innovation in digital university teaching by the Ministry of Innovation, Science and Research of the State of North RhineWestphalia, Germany, and Stifterverband, Germany during the genesis of this project.

The author would like to thank the reviewers whose constructive criticism helped to improve this paper as well as the project. 


\section{References}

Abelson, H., Adida, B., Linksvayer, M., \& Yergler, N. (2012). CC REL: The Creative Commons Rights Expression Language. In M. D. De Rosnay \& J. C. De Martin (Eds.), The digital public domain: Foundations for an open culture. Open Book Publishers. doi:10.11647/obp.0019.10

Auer, S., Khalili, A., \& Tarasowa, D. (2013). Crowd-sourced Open Courseware Authoring with SlideWiki.org. International Journal of Emerging Technologies in Learning (iJET), 8(1), 62-63. doi:10.3991/ijet.v8i1.2539

Chacon, S., \& Straub, B. (2014). Pro Git (2nd ed.). Apress. doi:10.1007/ 978-1-4842-0076-6

Falconer, I. J., Littlejohn, A., McGill, L., \& Beetham, H. (2016). Motives and tensions in the release of open educational resources: The UKOER program. Australasian Journal of Educational Technology, 32(4). doi:10.14742/ajet.2258

Hilton, J. I., Wiley, D., Stein, J., \& Johnson, A. (2010). The four 'R's of openness and ALMS analysis: frameworks for open educational resources. Open Learning, 25(1), 37-44. doi:10.1080/02680510903482132

Horrocks, I. (2008). Ontologies and the Semantic Web. Commun. ACM, 51(12), 58-67. doi:10.1145/1409360.1409377

Lechtenbörger, J. (2019a). Erstellung und Weiterentwicklung von Open Educational Ressources im Selbstversuch. MedienPädagogik, 34, 101-117. doi:10.21240/mpaed/34/ 2019.03.02.X

Lechtenbörger, J. (2019b). Simplifying license attribution for OER with emacs-reveal. In 17. Fachtagung Bildungstechnologien (DELFI 2019), accepted.

Novak, G. M. (2011). Just-in-time teaching. New Directions for Teaching and Learning, 2011(128), 63-73. doi:10.1002/tl.469

Ro, J. H., \& Namkoong, J.-E. (2019). Org-Coursepack: A Modular and Reusable Teaching Materials Template in Org-mode. Journal of Open Source Education, 2(8). doi:10.21105/ jose.00034

Rockley, A. (2001). The Impact of Single Sourcing and Technology. Technical Communication, 48(2), 189-193.

Schulte, E., \& Davison, D. (2011). Active Documents with Org-Mode. Computing in Science Engineering, 13(3), 66-73. doi:10.1109/MCSE.2011.41

UNESCO. (2017). Ljubljana OER Action Plan 2017. Retrieved April 14, 2019, from https://en.unesco.org/sites/default/files/ljubljana_oer_action_plan_2017.pdf

Wright, J. (2019). Beamer - A LaTeX class for producing presentations. Retrieved April 17, 2019, from https://github.com/josephwright/beamer 